\title{
Empowernder Wissenstransfer im Kontext von wissenschaftlicher Praxis und Gender
}

Mareen Hauke

Wissenstransfer ist ein Begriff, dessen Bedeutung sich wie jeder Begriff im alltäglichen und (fach)spezifischen Gebrauch bildet und verändert. Es hängt u.a. vom jeweiligen Kontext ab, was mit ,Wissenstransfer gemeint ist. Geistes-, Sozial- und Kulturwissenschaftler_innen* (GSK-Wissenschaftler_innen*) verstehen unter Wissenstransfer u.a. die Vermittlung von wissenschaftlichen Erkenntnissen an die Öffentlichkeit bzw. die Praxis sowie umgekehrt die Vermittlung von Alltags-, Anwendungs- und Expert_innenwissen aus der Öffentlichkeit und verschiedenen Praxisfeldern an die Wissenschaft (Wutti and Hayden 2017). Mit ,Wissenstransfer' wird in den GSK also meistens kein einseitiges, von den Universitäten geschaffenes, Wissensangebot an Dritte, sondern ein wechselseitiger Wissensaustausch verbunden. Im Kontext der Feministischen Wissenschaften/Gender Studies werden ähnliche Auffassungen zum Thema Wissenstransfer vertreten. Zwar wird der konkrete Begriff, Wissenstransfer' in der queer_feministischen ${ }^{1}$ Literatur bisher eher selten verwendet, die Bedeutung einer

1 Der Unterstrich oder 〈Gap〉 (von engl. gap = Lücke, Spalt, Kluft) zwischen 'queer` und (feministischen*) soll hier anzeigen, dass queere und feministische Ansätze sich sowohl widersprechen als auch ergänzen können. Ein Widerspruch besteht z.B. dann, wenn von einem Feminismus ausgegangen wird, der auf essentialistischen Grundannahmen beruht. Feministische Ansätze, die stattdessen von der sozialen Konstruktion von Geschlecht ausgehen und dies thematisieren 
Wechselbeziehung zwischen den konkreten Lebensrealitäten der Menschen* und den akademischen Diskursen bzw. zwischen ,Theorie und ,Praxis‘ wird jedoch häufig betont. Eine hohe Relevanz wird diesen Wechselbeziehungen vor allem für das Erreichen und Unterstützen emanzipatorischer Bestrebungen zugeschrieben (Althoff, Apel, Bereswill, Gruhlich, and Riegraf 2017; Fleisch 2013).

Gender ist ein aus dem Englischen übernommener Begriff, der übersetzt so viel wie «das soziale Geschlecht bedeutet. Gemeint ist, dass Geschlecht nicht einfach gegeben ist, sondern im Alltag durch Geschlechtszuschreibungen, durch Rollenerwartungen und das «Aufführen〉 von Geschlechterrollen sozial konstruiert wird (Butler 1991). Das sogenannte biologische Geschlecht (Engl.: 〈sex〉) stellt dabei eine besonders naturalisierte Form der sozialen Konstruktion von Geschlecht dar. Denn auch dieses «hat keine_r einfach, sondern es wird eine_r/eine_m anhand von bestimmten Körpermerkmalen (z.B.: Beschaffenheit der primären und sekundären Geschlechtsmerkmale, der Geschlechtschromosomen sowie des Mengenverhältnisses von Geschlechtshormonen) zugeschrieben (Butler 1991; Hornscheidt and Tudor 2011).

Textbox 1: Gender

Wissenschaftliche Diskurse können dafür kritisiert werden, dass sie teilweise nur für einen kleinen, relativ stark privilegierten Personenkreis - vorwiegend für das Fachpublikum - tatsächlich verständlich und damit nutz- und gestaltbar sind. Bestimmt werden diese Diskurse in erster Linie von Wissenschaftler_innen*, deren Handlungsspielraum durch verschiedene, teilweise festgefahrene, teilweise flexible Machtstrukturen beeinflusst wird. Im Rahmen dieses Handlungsspielraums hängt es von den Entscheidungen der Wissenschaftler_innen*

bzw. analysieren, ergänzen sich hingegen gut mit queerer Politik, die sich dadurch auszeichnet diskriminierenden Rekonstruktionsprozessen mit Dekonstruktionsprozessen zu begegnen (Bretz and Lantzsch 2013). 
ab, ob Anliegen und Denkweisen aus diskriminierten Positionen wissenschaftliche Aufmerksamkeit geschenkt wird oder nicht. Ebenso entscheiden die einzelnen Mitglieder der Scientific Community darüber, für wen sie ihre Wissensproduktionen zugänglich gestalten. Wie, für wen und mit wem geforscht wird, ist darüber hinaus auch disziplin- und ressourcenabhängig. Im universitären Wissenschaftsbetrieb sind nur wenige Ressourcen dafür vorgesehen wissenschaftliche Erkenntnisse so aufzubereiten, dass sie für Laien verständlich und nutzbar werden (Hayden, Weiß, Pechriggl, and Wutti 2018; Wutti and Hayden 2017). Wer eine wissenschaftliche Karriere anstrebt, ist vor allem gefordert, sich innerhalb der Scientific Community einen $\mathrm{Na}$ men zu machen. Wissenstransferleistungen jenseits von ,Science to Science ' werden selten gewürdigt und entsprechend oft ehrenamtlich realisiert (siehe Abschnitt 2.3 in diesem Issue, bzw. Hayden et al. 2018).

Der Begriff ‘Empowerment〉 wurde aus dem Englischen ins Deutsche übernommen und bedeutet übersetzt etwa «Selbstermächtigung` oder «Stärkung der Eigenkräfte“. Er findet sowohl in der psychosozialen Praxis als auch in sozialen Bewegungen, wie z.B. in den Bürgerrechts-, Frauen*- und Selbsthilfebewegungen, Anwendung (Schwarz 2014). Ziel ist die Erweiterung des Handlungsspielraums von gesellschaftlich Benachteiligten*. Dafür muss auf verschiedenen, zusammenwirkenden Ebenen angesetzt werden, wie z.B. auf der Ebene des sozialen Handelns, der Gemeindeentwicklung, der Grundlagenforschung und auf rechtlicher Ebene (Collins 1991; Schwarz 2014).

Textbox 2: Empowerment

In den Feministischen Wissenschaften/Gender Studies besteht häufig der Anspruch, Wissenstransferleistungen zu erbringen, die hilfreich für Menschen* sind, die insbesondere im Kontext der Kategorie ,Geschlecht' struktureller Diskriminierung ausgesetzt sind. Tatsächlich 
sind diese Wissenschaftsbereiche bereits aus demselben Anspruch heraus entstanden. So stand von Anfang an die Kritik an androzentrischen Traditionen und Strukturen sowohl in der Wissenschaft als auch in der Gesamtgesellschaft im Fokus. Feministische Wissenschaft verstand sich als explizit politisches Emanzipationsprojekt. Trotz verschiedener Kontroversen über Theorien und Methodik sowie über Ein- und Ausschlüsse innerhalb dieses Projekts, ist der Anspruch, anschlussfähige Forschung zu betreiben, nach wie vor aktuell (Althoff et al. 2017). Eine Möglichkeit, Forschung anschlussfähig zu machen besteht darin, neben den Forschungszielen auch die Machtstrukturen, die das Forschungsprojekt bestimmen, möglichst transparent zu machen. Dies kann durch die sogenannte ,kritische Verortung' (Tudor 2011) gewährleistet werden - ein Konzept, dem einige theoretische Vorannahmen zugrunde liegen: Es wird davon ausgegangen, dass Privilegierungen und Diskriminierungen soziale Konstruktionen sind, die durch ständiges Wiederholen immer wieder rekonstruiert werden. Durch diese Prozesse wird jedoch keine Situation 1:1 reproduziert, sondern es ergeben sich immer wieder kleine Änderungen (Haraway 1995). In diesen liegt das Potenzial zu großen Veränderungen, was sowohl als Chance als auch als Gefahr gesehen werden kann. Jegliche Veränderungen, so auch strukturelle, würden sich durch Intra-Aktionen $^{2}$ innerhalb eines Strukturnetzwerks ergeben, in dem sich alles auch jeder Mensch* - bewege und zugleich von ihm bewegt werde

\footnotetext{
${ }^{2}$ Der Begriff der Intra-Aktion soll zweierlei verdeutlichen: Einerseits gilt die Annahme, dass es kein ‘Außen` des bewegten Strukturnetzwerks gibt; alles entsteht und vergeht innerhalb desselben und es gibt entsprechend keine Einflussmöglichkeit oder Betrachtungsweise von ‘außen». Andererseits veranschaulicht der Begriff die Beweglichkeit der Grenzen innerhalb des Netzwerks. So interagieren Dinge und Menschen* nicht mit- oder untereinander, sondern sie intraagieren. Nach jeder Intra-Aktion sind sie verändert, wenn auch nur geringfügig (Barad 2003).
} 
(Barad 2003). Unter kritischer Verortung wird die explizite Benennung und Reflexion möglichst vieler, für das Forschungsprojekt relevanter Positionen und Interdependenzen innerhalb dieses Netzwerks verstanden sowie das Transparentmachen von Zielen und Zielgruppen. Zur kritischen Verortung gehört daher einerseits offenzulegen, von welchen Privilegierungen und Diskriminierungen die Forschenden selbst betroffen sind (Tudor 2011). Andererseits sollte auch die Forschungssituation als ganze unter die Lupe genommen werden (Clarke 2012). Warum, von wem und für wen wird das Projekt finanziert? Wer darf bei diesem Projekt mitwirken und wer nicht? Wem nutzt es und wem nicht? (Schwarz 2013). Wer sich als Forscher_in* diese Fragen stellt, bekommt relativ schnell einen Überblick vom eigenen Handlungsspielraum und darüber, wo Gefahr besteht, diskriminierte Standpunkte zu übersehen, zu überhören, nicht wahrzunehmen und entsprechend $\mathrm{zu}$ versäumen, sie in die Forschung miteinzubeziehen. Diese Gefahr besteht vor allem dort, wo der_die Forscher_in* selbst privilegiert ist und daher das Wissen, das sich aus diskriminierten Positionen heraus ergibt, nicht kennt und auch nur bedingt nachvollziehen kann, wie sich das Leben in diesen Positionen gestaltet (Haraway 1995). Gegebenenfalls empfiehlt es sich, Menschen* aus diskriminierten Positionen selbst aktiv am Forschungsprozess teilhaben zu lassen und ihnen damit den nötigen Gestaltungsspielraum zu geben, der verhindert, dass diskriminierende Handlungen, z.B. in Form von Zuschreibungen oder „für jemanden sprechen" wiederholt werden. Kritische Verortung ist im Forschungskontext also der Versuch, dem Anspruch verantwortungsbewussten wissenschaftlichen Arbeitens gerecht zu werden, indem Machtverhältnisse reflektiert und transparent gemacht werden und die Forschung entsprechend auch danach ausgerichtet wird, Diskriminierungen möglichst nicht zu reproduzieren, sondern ihnen im besten Fall aktiv entgegenzuwirken (Tudor 2011). Empowernde Wissenstransferpro- 
zesse können durch kritisch verortete Forschung initiiert werden, indem die Forschung buchstäblich angreifbar wird - einerseits durch das Transparentmachen von Machtverhältnissen und Zielen und andererseits durch das Einräumen von Teilhabemöglichkeiten für gesellschaftlich Benachteiligte*. Diesem Anspruch versucht auch die laufende Forschung gerecht $\mathrm{zu}$ werden, indem empowernder Wissenstransfer nicht nur als Forschungsgegenstand betrachtet wird, sondern Forschungsprozess und -ergebnisse idealerweise selbst zum Empowerment benachteiligter Menschen* beitragen sollen. Der Fokus liegt dabei auf den vielfältigen Bereichen des empowernden Wissenstransfers, die im Kontext von Diskriminierungen aufgrund von Geschlecht/Gender stattfinden. Im nachfolgenden Abschnitt wird die diesbezüglich durchgeführte Forschung vorgestellt und diskutiert. 


\section{Literatur}

Althoff, Martina, Magdalena Apel, Mechthild Bereswill, Julia Gruhlich and Birgit Riegraf, eds. 2017. Feministische Methodologien und Methoden - Traditionen, Konzepte, Erörterungen. Wiesbaden: Springer.

Barad, Karen. 2003. "Posthumanist Performativity: Toward an Understanding of How Matter Comes to Matter." Signs, 28(3) (Spring): 801-831. https://doi.org/10.1086/345321.

Bretz, Leah, and Nadine Lantzsch. 2013. Queer_Feminismus. Label \& Lebensrealität. Münster: Unrast.

Butler, Judith. 1991. Das Unbehagen der Geschlechter. Frankfurt am Main: Suhrkamp.

Clarke, Adele E. 2012. Situationsanalyse. Grounded Theory nach dem Postmodern Turn. Wiesbaden: Springer.

Fleisch, Renate. 2013. "Über die unerlässliche Verbindung von feministischer Theorie mit feministischer Praxis." In Prekarität und Freiheit? Feministische Wissenschaft, Kulturkritik und Selbstorganisation, edited by Dagmar Fink, Birge Krondorfer, Sabine Prokop, and Claudia Brunner, 169-177. Münster: Westfälisches Dampfboot.

Hayden, Markus C., Weiß, Martin, Pechriggl, Alice, and Daniel Wutti. 2018. "Insights into University Knowledge Transfer in the Social Sciences and Humanities (SSH) and Other Scientific Disciplines - More Similarities than Differences." Frontiers in Research Metrics and Analytics 3, 32. https://doi.org/10.3389/frma.2018.00032 
Haraway, Donna. 1995. "Situiertes Wissen.” In Die Neuerfindung der Natur. Primaten, Cyborgs und Frauen, edited by Carmen Hammer, and Immanuel Stieß, 73-97. Frankfurt am Main: Campus.

Hill Collins, Patricia. 1991. "Knowledge, Consciousness, and the Politics of Empowerment." In Black Feminist Thought. Knowledge, Consciousness, and the Politics of Empowerment, edited by Patricia Hill Collins, 2. Edition, 221-238. London: Routledge.

Hornscheidt, Lann, and Alyosxa Tudor, 2011. "Feminismus." In Feminismus schreiben lernen, edited by AK Feministische Sprachpraxis, 12-56. Frankfurt am Main: Brandes \& Aspel.

Schwarz, Silke. 2013. "Intersectionality traveling into psychology." Portal Intersektionalität. Forschungsplattform und Praxisforum für Intersektionalität und Interdependenzen, http://portalintersektionalitaet.de/theoriebildung/ueberblickstexte/schwarz/.

Schwarz, Silke. 2014. Gendergerechtigkeit als Universalkonzept? Kritische und kultursensible Analysen von Gendermainstreaming nach einer Katastrophe. Wiesbaden: Springer.

Tudor, Alyosxa. 2011. "feminismus w_orten lernen. Praktiken kritischer Ver_Ortung in feministischen Wissensproduktionen." In Feminismus schreiben lernen, edited by AK Feministische Sprachpraxis, 57-99. Frankfurt am Main: Brandes \& Aspel.

Wutti, Daniel, and Markus Hayden. 2017. "Knowledge Transfer in the Social Sciences and Humanities (SSH) - Definition, Motivators, Obstacles, and Visions." Colloquium: New Philologies, 2(1): 87-101. https://doi.org/10.23963/cnp.2017.2.1.7 